\title{
Mitochondrial separation protein inhibitor inhibits cell apoptosis in rat lungs during intermittent hypoxia
}

\author{
DAN ZHAO ${ }^{1,2^{*}}$, CHEN-YI YIN ${ }^{3 *}$, XIAN-WEI YE ${ }^{1 *}$, ZI-FEN WAN $^{1}$, DE-GANG ZHAO ${ }^{2,4}$ and XIANG-YAN ZHANG ${ }^{1}$ \\ ${ }^{1}$ Department of Respiratory and Critical Care Medicine, Guizhou Provincial People's Hospital, Guiyang, Guizhou 550002; \\ ${ }^{2}$ Life Sciences College of Guizhou University, Guiyang, Guizhou 550025; ${ }^{3}$ Department of Graduate School, \\ Zunyi Medical University, Zunyi, Guizhou 563000; ${ }^{4}$ The Key Laboratory of Plant Resources Conservation and Germplasm Innovation \\ in Mountainous Region (Ministry of Education), Guizhou University, Guiyang, Guizhou 550025, P.R. China
}

Received April 23,2018; Accepted November 22, 2018

DOI: 10.3892/etm.2019.7201

\begin{abstract}
Obstructive sleep apnoea (OSA) is a very common sleep and breathing disorder that occurs in worldwide. It is important to develop a more effective treatment for OSA to overcome lung cell apoptosis during intermittent hypoxia (IH). A mitochondrial separation protein inhibitor (Mdivi-1) has been demonstrated to be a powerful tool for inhibiting apoptosis. In the present study, the protective effect and possible mechanism of apoptosis in lung cells during IH was investigated using in vivo and in vitro experiments. Following IH exposure for 4 weeks, the lung tissues of Sprague Dawley rats exhibited interstitial lesions, while Mdivi-1 reduced these pulmonary interstitial lesions. B-cell lymphoma (Bcl)-2 mRNA and protein expression levels were decreased however caspase-3, caspase-9 and dynamin-related protein 1 (Drp-1) mRNA and protein expression levels were increased. Following Mdivi-1 intervention, Bcl-2 mRNA and protein expression levels were increased while caspase-3, caspase-9 and Drp-1 mRNA and protein expression levels were decreased $(\mathrm{P}<0.05)$. After exposure to IH for $12 \mathrm{~h}$, the apoptosis rate of WTRL1 cells in rats increased gradually with the IH time $(\mathrm{P}<0.05)$. Bcl-2 mRNA and protein expression levels were decreased, whereas caspase-3, caspase-9, cytochrome C (Cyt-C) and Drp-1 mRNA levels were increased, and caspase-3, caspase-9 and Drp-1 protein expression levels were increased. After Mdivi-1 intervention, Bcl-2 mRNA and protein expression levels were increased but caspase-3, caspase-9, Cyt-C and Drp-1 mRNA levels were decreased
\end{abstract}

Correspondence to: Professor Xiang-Yan Zhang, Department of Respiratory and Critical Care Medicine, Guizhou Provincial People's Hospital, 83 ZhongShan East Road, Guiyang, Guizhou 550002, P.R. China

E-mail: zxy35762@126.com

*Contributed equally

Key words: mitochondrial division inhibitor-1, lung, cell apoptosis, intermittent hypoxia, mitochondrial signalling pathways along with caspase-9, Cyt-C and Drp-1 protein expression levels which were decreased $(\mathrm{P}<0.05)$. The results of the present study suggest that Mdivi-1 may be a potential agent for treating OSA because it inhibits the mitochondrial pathway and reduces apoptosis.

\section{Introduction}

Obstructive sleep apnoea (OSA) is a very common sleep and breathing disorder that is characterized by the repetitive collapse of the upper airway during sleep (1); in addition, OSA has high morbidity in paediatric and adult populations. In OSA, upper airway obstruction is usually associated with recurrent hypoxemia interspersed with periods of reoxygenation and is typically terminated by brief arousals, resulting in intermittent hypoxia (IH) and sleep fragmentation (2). The oscillatory nature of $\mathrm{IH}$, especially chronic IH, may mimic the paradigm of ischaemia-reperfusion in that tissues and cells are exposed to periods of low and high $\mathrm{O}_{2}$, which may lead to oxidative stress and induces lipid peroxidation, apoptosis, stress-responsive proteins, cell death and inflammation (3-5). Studies have confirmed that IH via different signalling pathways induces cell apoptosis $(6,7)$. Mitochondrial separation protein inhibitor (Mdivi-1) is a selective dynamin-related protein 1 (Drp-1) inhibitor that has been reported to be a potential therapeutic target for brain, cardiovascular, liver and tumour angiogenesis $(8,9)$. How Mdivi-1 inhibits lung apoptosis during IH remains unclear. The aim of the present study was to investigate the protective effect and possible mechanism of Mdivi-1 in lung cell apoptosis during IH.

\section{Materials and methods}

Animals. A total of 32 specific pathogen free 6-8-week-old male Sprague Dawley (SD) rats (body weight, 180-220 g) were purchased from the Animal Care and Use Committee of the Army Medical University (Chongqing, China). The experiments were conducted in accordance with the Guide for the Care and Use of Laboratory Animals (10). All experimental procedures were approved by the Ethics Committee of Guizhou Provincial People's Hospital (Guiyang, China). All 
efforts were made to minimize animal suffering and to reduce the number of animals used.

In vivo experimental design. All animals were maintained in a controlled environment $\left(22-25^{\circ} \mathrm{C}, 40-60 \%\right.$ relative humidity, with a 12-h light-dark cycle with the lights on at 08:00 am) with food and water available. Animals were allowed to acclimatize for 1 week to the experimental chamber. The rats were allocated randomly to four groups: Control group $(\mathrm{n}=8)$, IH group $(\mathrm{n}=8), \mathrm{IH}+25 \mathrm{mg} / \mathrm{kg}$ Mdivi-1 group $(\mathrm{n}=8)$ and $\mathrm{IH}+50 \mathrm{mg} / \mathrm{kg}$ Mdivi-1 group $(\mathrm{n}=8)$. The rats in the IH group, $\mathrm{IH}+25 \mathrm{mg} / \mathrm{kg}$ Mdivi-1 group and IH+50 mg/kg Mdivi-1 group were exposed to $\mathrm{IH}$ conditions $\left(21 \% \mathrm{O}_{2}, 5 \% \mathrm{CO}_{2}\right.$ and balanced $\mathrm{N}_{2}$ ) for $90 \mathrm{sec}$ and hypoxia $\left(8 \% \mathrm{O}_{2}, 5 \% \mathrm{CO}_{2}\right.$ and balanced $\mathrm{N}_{2}$ for $30 \mathrm{sec}$ ) from 08:00 am to 04:00 pm daily for 4 consecutive weeks (11-13). The chambers had a rear $\mathrm{N}_{2}$ inlet and a front air extractor, which enabled recovery to normoxia. A computerized system controlled the valve inlets and the alternating cycles of the extractors. The $\mathrm{CO}_{2}$ in the chamber was maintained at a low level by continuous air extraction (14). Rats in the control group were maintained under normoxic conditions during the experiment. The treatment groups were injected intraperitoneally with Mdivi-1 (MedChemExpress, Monmouth Junction, NJ, USA) prior to oxygen treatment every day (15). A general clinical pain score for scoring cyanosis condition for decisions about endpoint was use as presented in Table I (16).

Sample collection and tissue preparation. Trained personnel euthanized the experimental animals via $\mathrm{CO}_{2}$ inhalation following IH exposure. Rats were left in the chamber until clinical death could be ensured. The flow rate of $\mathrm{CO}_{2}$ displaced $20 \%$ of the volume chamber per minute. If no breath was seen for $60 \mathrm{sec}$ the rodent was removed from the chamber. To ensure death, the rat's chest was felt to determine that there is no longer a heartbeat. Death was verified following euthanasia and prior to disposal. The lungs were dissected out and the right lower lobes were fixed in $4 \%$ paraformaldehyde at room temperature for $48 \mathrm{~h}$. The remaining lung tissues were frozen in liquid nitrogen and then stored at $-80^{\circ} \mathrm{C}$ until further analysis.

Histological examination. The right lower lobes were fixed in $4 \%$ paraformaldehyde at room temperature for $24 \mathrm{~h}$, embedded in paraffin blocks and slices into $6-\mu \mathrm{m}$-thick sections. The sections were then stained with haematoxylin for $5 \mathrm{~min}$ and eosin for $90 \mathrm{sec}$ at room temperature, and observed by light microscopy. Damage to the lung tissue was scored by the pathologist on a scale of 1 (no injury) to 4 (worst), as described previously (17).

In vitro experimental design. WTRL1 cells (a rat pulmonary alveolar epithelial cell line; http://www.cellresource. cn/fdetail.aspx?id=1349) were purchased from the National Infrastructure of Cell Line Resource, (Beijing, China). The cells were grown in RPMI-1640 medium (HyClone, Logan, UT, USA) with 10\% FBS (Gibco; Thermo Fisher Scientific, Inc., Waltham, MA, USA), penicillin (100 units/ml) and streptomycin $(100 \mu \mathrm{g} / \mathrm{ml})$ and were cultured at $37^{\circ} \mathrm{C}$ in $5 \% \mathrm{CO}_{2}$ in a humidified incubator. WTRL1 cells in the logarithmic growth phase were divided randomly into five groups $\left(3 \times 10^{6}\right.$ cells/well) in 6-well plates: Control, $\mathrm{IH}$, $\mathrm{IH}+10 \mu \mathrm{M}$ Mdivi-1, IH+50 $\mu \mathrm{M}$ Mdivi-1 and $\mathrm{IH}+100 \mu \mathrm{M}$ Mdivi-1. The control group was exposed to air, while the IH group, IH+10 $\mu \mathrm{M}$ Mdivi-1 group, $\mathrm{IH}+50 \mu \mathrm{M}$ Mdivi-1 group and $\mathrm{IH}+100 \mu \mathrm{M}$ Mdivi-1 group were placed in a low oxygen culture box containing $5 \% \mathrm{O}_{2}$ for $1 \mathrm{~h}$. The box was then reoxygenated with $21 \% \mathrm{O}_{2}$ for $30 \mathrm{~min}$ and this process was repeated for $12 \mathrm{~h}$. The treatment groups were incubated with Mdivi-1 for $30 \mathrm{~min}$ prior to IH culture.

Flow cytometry. WTRL1 cells were grown logarithmically and placed on $60-\mathrm{mm}$ Petri dishes in a low oxygen culture box containing $5 \% \mathrm{O}_{2}$ for $1 \mathrm{~h}$. The box was reoxygenated with $21 \% \mathrm{O}_{2}$ for $30 \mathrm{~min}$ and this process was repeated for $6,12,24$ and $48 \mathrm{~h}$. Next, the cells were resuspended in Annexin V-PI from a dual-staining assay kit (Nanjing KeyGen Biotech. Co. Ltd., Nanjing, China) for $10 \mathrm{~min}$ at room temperature. The fluorescence signals from Annexin V and PI were measured by flow cytometry with a FACSCalibur ${ }^{\mathrm{TM}}$ flow cytometer (BD Bioscience), and the data were analysed using Cell Quest Pro software (version 5.0; BD Bioscience). The flow cytometry results were measured in $1 \mathrm{~h}$.

Reverse transcription-quantitative polymerase chain reaction $(R T-q P C R)$. Total RNA was extracted from lung tissues or cells with TRIzol (Thermo Fisher Scientific, Inc.). The D260/D280 ratio was measured and samples with a ratio between 1.8 and 2.0 were used for reverse transcription with a High Capacity cDNA Archive kit (Bio-Rad Laboratories, Inc., Hercules, CA, USA). The reverse transcription protocol were as follows: $25^{\circ} \mathrm{C}$ for $10 \mathrm{~min}, 37^{\circ} \mathrm{C}$ for $120 \mathrm{~min}$ and $85^{\circ} \mathrm{C}$ for $5 \mathrm{~min}$. qPCR was applied to analyze the cDNAs by using the 2X Maxima SYBR-Green/ROX qPCR Master Mix kit (Thermo Fisher Scientific, Inc.) following the manufacturer's protocol. The amplification conditions were as follows: $95^{\circ} \mathrm{C}$ for $10 \mathrm{~min}$ for denaturation, followed by 40 cycles of $95^{\circ} \mathrm{C}$ for $15 \mathrm{sec}, 60^{\circ} \mathrm{C}$ for $30 \mathrm{sec}$ and $72^{\circ} \mathrm{C}$ for $60 \mathrm{sec}$. qPCR for Bcl-2, caspase-3, caspase-9, Cyt-C and Drp-1 was performed using TaqMan gene expression assays and the $2^{-\Delta \Delta \mathrm{Cq}}$ method with GAPDH as the endogenous control (18). The primers were synthesized by Sheng Gong (Shanghai) Co., Ltd. (Shanghai, China) and the sequences are presented in Table II.

Western blot analysis. Total protein cell lysates were isolated from rat lungs or from cells as described previously (19). Protein quantification was assessed using a bicinchoninic acid protein quantification assay and absorbance read at $562 \mathrm{~nm}$ was used to determine sample volumes for further analysis. A total of $50 \mu \mathrm{g}$ total protein from each tissue homogenate was separated by $30 \%$ SDS-PAGE and then transferred to polyvinylidene difluoride membranes, which were blocked with $5 \%$ skim milk at room temperature for $1 \mathrm{~h}$. Membranes were washed twice with TBST and incubated with primary antibodies at $4{ }^{\circ} \mathrm{C}$ overnight against Bcl-2 polyclonal antibody (cat. no. YT0470), caspase-3 polyclonal antibody (cat. no. YT0656), caspase-9 polyclonal antibody (cat. no. YT0662), Drp1 Polyclonal antibody (cat. no. YT1414; all 1:1,000; ImmunoWay Biotechnology Company, Plano, TX, USA), Cyt-C antibody 
Table I. Assessing pain and distress in animal.

1) No indication of pain and distress

2) Mild or anticipated pain and distress

3) Moderate pain and distress

4) Severe pain and distress
Normal; well groomed; alert; active; good condition; asleep or calm; normal appetite; $\mathrm{BCS}=3,4$ or 5

Not well groomed; awkward gait; slightly hunched; looks at wound or pulls away when area touched; mildly agitated; $\mathrm{BCS}=2$

Rough hair coat; dirty incision; squinted eyes; moves slowly; walks hunched and/or slowly; depressed or moderately agitated; slight dehydration; pruritic; restless; uncomfortable; not eating or drinking; $\mathrm{BCS}=2$.

Very rough hair coat; eyes sunken (severe dehydration); slow to move or non-responsive when coaxed; hunched; large abdominal mass; dyspnea; self mutilating; violent reaction to stimuli or when approached; $\mathrm{BCS}=1$

BCS, body condition score.

Table II. Primer sequences for reverse transcription-quantitative polymerase chain reaction.

Primer name

Primer sequence (5'-3')

\section{GAPDH}

B-cell lymphoma 2

Caspase-3

Caspase-9

Cytochrome C

Dynamin-related protein 1

F: AGCCACATCGCTCAGACAC
R: GCCCAATACGACCAATCC
F: TCCTTCCAGCCTGAGAGCAACC
R: TCACGACGGTAGCGACGAGAG
F: GTAGCAGTGTGCCGCTGTGTC
R: ACTCTTGTCCATCGCCTCTCCTC
F: ATGGAGGAGGCTGACCGGCAACTCCTG
R: TCATGAAGTTTTAAAGAACAGCTTCTTC
F: AGGAGGCAAGCATAAGAC
R: ATTAGGTCTGCCCTTTCT
F: TTCGCCGCCTGGAGGACC
R: CACTTCGTTGCCACAATGCTGAA

F: AGCCACATCGCTCAGACAC

F: TCCTTCCAGCCTGAGAGCAACC

R: TCACGACGGTAGCGACGAGAG

R: TCATGAAGTTTTAAAGAACAGCTTCTTC

F: AGGAGGCAAGCATAAGAC

F: TTCGCCGCCTGGAGGACC

R: CACTTCGTTGCCACAATGCTGAA

F, forward; R, reverse.

(cat. no. 10993-1-AP; 1:1,000; ProteinTech, Group, Inc., Chicago, IL, USA), mouse monoclonal $\beta$-actin antibody (1:4,000; cat. no. YM3138; ImmunoWay Biotechnology Company). Membranes were washed twice and incubated with horseradish peroxidase-conjugated goat anti-mouse IgG (1:6,000; cat. no. PMK-014-091; Bioprimacy, Wuhan, China) and goat anti-rabbit IgG (1:8,000; cat. no. PMK-014-090; Bioprimacy, Wuhan, China) for $2 \mathrm{~h}$ at room temperature. The blots were visualized with eECL western blot kit (Beyotime Institute of Biotechnology, Jiangsu, China). The intensities of the bands were measured using ImageLab 5.1 Software (Bio-Rad Laboratories, Inc.). Rat $\beta$-actin was used as the loading control in western blotting.

Statistical analysis. All experiments were repeated three times. All data are presented as mean \pm standard deviation. Comparisons of two or three experimental conditions were evaluated using an unpaired Student's t-test or one-way analysis of variance and the Bonferroni t-test followed by the Bonferroni post-test using GraphPad Prism 5.0 software (GraphPad Software Corp, San Diego, CA, USA).
$\mathrm{P}<0.05$ was considered to indicate a statistically significant difference.

\section{Results}

Lung histopathology. Histopathological examinations were performed for the lungs of rats subjected to normoxia, IH, $\mathrm{IH}+25 \mathrm{mg} / \mathrm{kg}$ Mdivi-1 and IH+50 mg/kg Mdivi-1. IH caused severe interstitial pneumonia and a large number of inflammatory cells, while following Mdivi-1 intervention, interstitial pneumonia and inflammatory cells were reduced. The effect in the IH+50 mg/kg Mdivi-1 was more obvious compared with in the IH+25 mg/kg Mdivi-1 group (Fig. 1). Consistent with these histopathological observations, the lung injury score in the IH group was significantly increased compared with the control group $(\mathrm{P}<0.005)$ and the score in the $\mathrm{IH}+50 \mathrm{mg} / \mathrm{kg}$ Mdivi-1 group was significantly decreased compared with in the IH group $(\mathrm{P}<0.005)$.

Detection of Bcl-2, caspase-3, caspase-9, Cyt-C and Drp-1 expression levels via $R T-q P C R$. After SD rats were exposed 
A

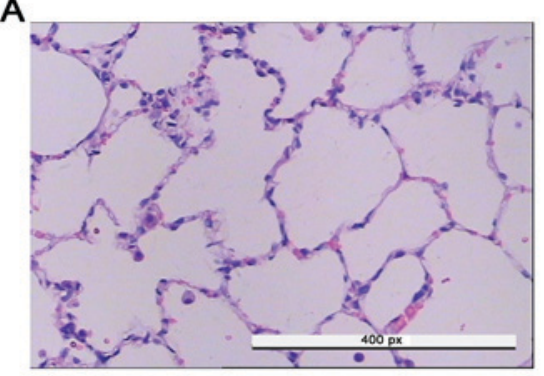

C

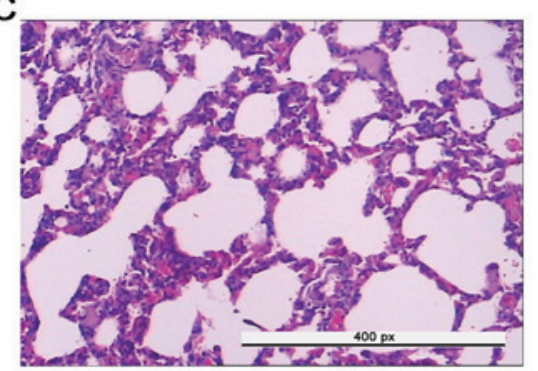

B

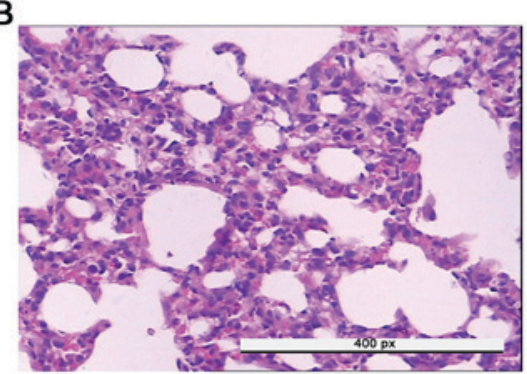

D

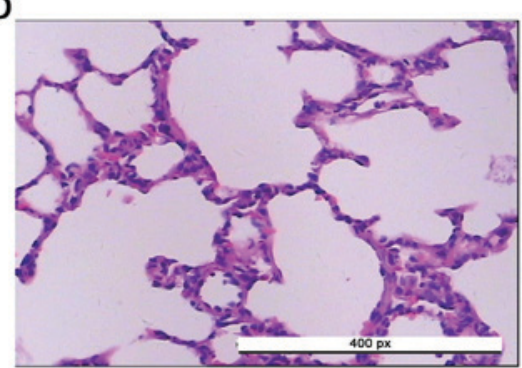

$\mathrm{E}$

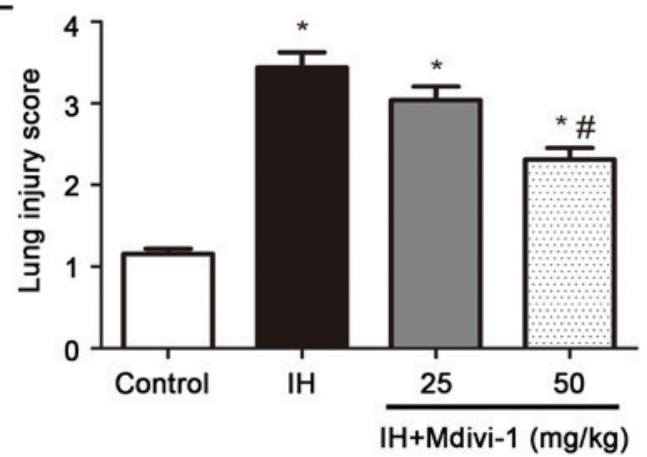

Figure 1. Detection of variations in the lung media of rats in each group using haematoxylin and eosin staining (magnification, 400 ; scale bar, $50 \mu \mathrm{m}$ ). (A) The control group, $\mathrm{n}=8$. (B) The IH group, $\mathrm{n}=8$. (C) The $\mathrm{IH}+25 \mathrm{mg} / \mathrm{kg}$ Mdivi-1 group, $\mathrm{n}=8$. (D) $\mathrm{IH}+50 \mathrm{mg} / \mathrm{kg}$ Mdivi-1 group, $\mathrm{n}=8$. (E) Compared with normoxia, IH caused interstitial alterations in the lung tissues of SD rats, while Mdivi-1 significantly reduced the pulmonary interstitial lesions. The effects in the IH+50 mg/kg Mdivi-1 group were more obvious than those in the IH+25 mg/kg Mdivi-1 group. IH, intermittent hypoxia; SD, Sprague Dawley; Mdivi, mitochondrial division inhibitor- 1 . " $\mathrm{P}<0.05$ vs. group $\mathrm{C}$; " $\mathrm{P}<0.05$ vs. group IH.

to IH for 4 weeks and after WTRL1 cells were exposed to $\mathrm{IH}$ for $12 \mathrm{~h}$ in culture, the expression of apoptosis markers was increased at the gene level in the rat lung tissue cells and WRTL1 cells. In addition, Mdivi-1 treatment effectively reduced the apoptosis rate of the hypoxia-exposed cells. After SD rats were exposed to $\mathrm{IH}$ for 4 weeks, Bcl-2 mRNA levels were significantly decreased $(\mathrm{P}<0.05$; Fig. $2 \mathrm{~A})$, but caspase-3 and caspase- 9 mRNA levels were significantly increased compared with the control group rats $(\mathrm{P}<0.05 ;$ Fig. $2 \mathrm{~B}$ and $\mathrm{C})$. After Mdivi-1 intervention, Bcl-2 mRNA levels were significantly increased and caspase-3, and Drp-1 mRNA levels were significantly decreased in the $\mathrm{IH}+50 \mathrm{mg} / \mathrm{kg}$ Mdivi-1 group compared with the IH group $(\mathrm{P}<0.05$; Fig. $2 \mathrm{~A}, \mathrm{~B}$ and $\mathrm{D})$. However, no significant difference in Cyt $\mathrm{C}$ levels was identified between the groups (Fig. 2E). After WTRL1 cells were exposed to IH for $12 \mathrm{~h}, \mathrm{Bcl}-2 \mathrm{mRNA}$ levels were significantly decreased, but caspase-3, caspase-9, Cyt-C and Drp-1 mRNA levels were significantly increased compared with the control group ( $\mathrm{P}<0.05$; Fig. 3A-E). After Mdivi-1 intervention, Bcl-2 mRNA levels were significantly increased and caspase-3, caspase-9, Cyt-C and Drp-1 mRNA levels were significantly decreased compared with in the IH group $(\mathrm{P}<0.05)$.
Western blotting results for Bcl-2, caspase-3, caspase-9, Drp-1 and Cyt-C. After SD rats were exposed to IH for 4 weeks and after WTRL1 cells were exposed to IH for $12 \mathrm{~h}$ in culture, the expression of apoptosis markers was increased at the protein level in rat lung tissue cells and WRTL1 cells. In addition, Mdivi-1 treatment reduced the apoptosis rate of the hypoxia-exposed cells. Western blotting results for Bcl-2, caspase-3, caspase-9, Cyt-C and Drp-1 protein expression in rat lungs are presented in Fig. 4. After SD rats were exposed to IH for 4 weeks, the protein expression of Bcl-2 in the IH group was significantly decreased, but the protein expression of caspase-3, caspase-9 and Drp-1 was significantly increased compared with the control group $(\mathrm{P}<0.05$; Fig. 4A-E). After Mdivi-1 intervention, the protein expression of Bcl-2 was significantly increased and the protein expression of caspase-3, caspase-9, and Drp-1 was significantly decreased in the $\mathrm{IH}+50 \mathrm{mg} / \mathrm{kg}$ Mdivi-1 group compared with the $\mathrm{IH}$ group $(\mathrm{P}<0.05)$. After WTRL1 cells were exposed to IH for $12 \mathrm{~h}, \mathrm{Bcl}-2$ protein expression was significantly decreased in the IH group and caspase- 3 , caspase- 9 and Drp- 1 protein expression was significantly increased $(\mathrm{P}<0.05$; Fig. 5A-E). After Mdivi-1 intervention, Bcl-2 protein expression was 
A

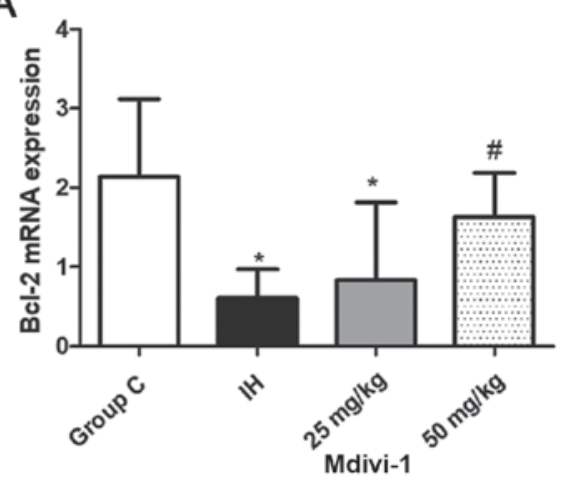

C.

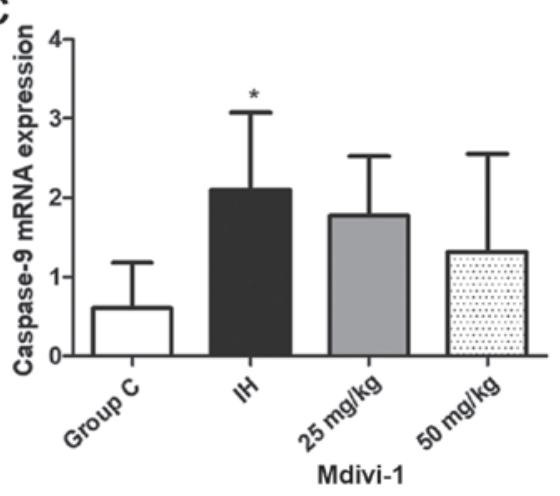

B

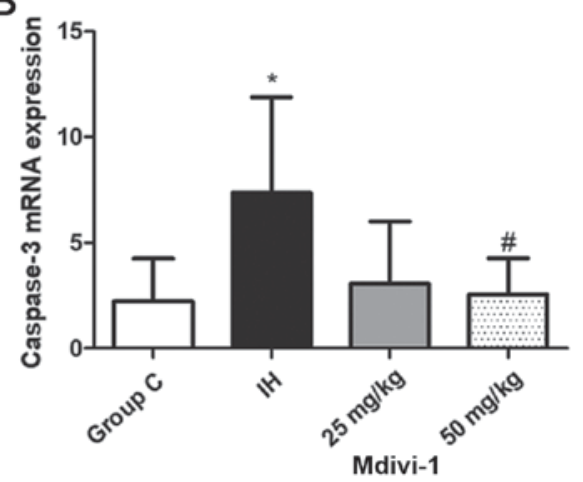

D

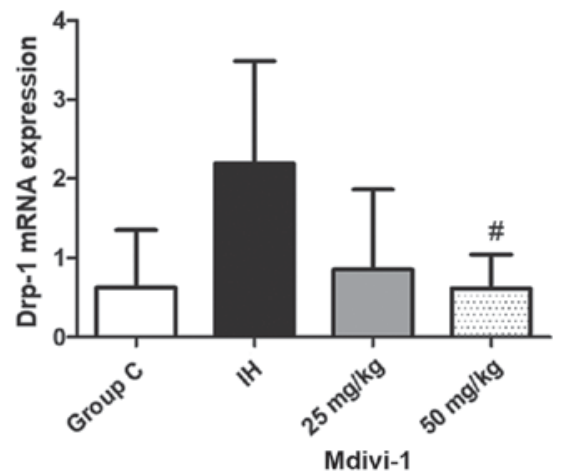

E

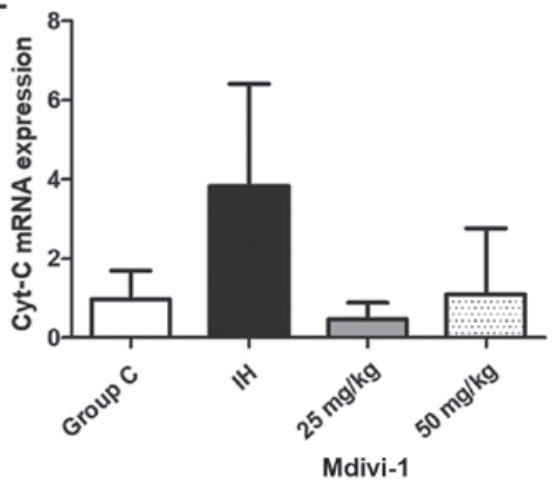

Figure 2. Detection of Bcl-2, caspase-3, caspase-9, Drp-1 and Cyt-C expression levels in rats lungs via reverse transcription-quantitative polymerase chain reaction. (A) Bcl-2 levels. (B) Caspase-3 levels. (C) Caspase-9 levels. (D) Drp-1 levels. (E) Cyt-C levels. Compared with those of group C, Bcl-2 mRNA levels were decreased, but caspase-3 and caspase- 9 mRNA levels were increased in the IH group, and these differences were statistically significant * $\mathrm{P}<0.05$ vs. group $\mathrm{C}$. Compared with those of the IH group, Bcl-2 mRNA levels were increased and caspase- 3 and Drp-1 mRNA levels were decreased in the IH+50 mg/kg Mdivi-1 group, ${ }^{\text {P }}<0.05$ vs. the IH group. IH, intermittent hypoxia; Mdivi, mitochondrial division inhibitor-1; Bcl, B-cell lymphoma; Cyt-c, cytochrome C; group C, control group.

significantly increased and caspase-9, Cyt-C, and Drp-1 protein expression was significantly decreased in the IH+100 $\mu$ M Mdivi-1 group (P<0.05; Fig. 5 A, B and D-F).

Detection of apoptosis levels. The apoptosis rate of WTRL1 cells was positively correlated with the time of exposure to IH. After WTRL1 cell exposure to IH for 6, 12, 24 and $48 \mathrm{~h}$, the rate of apoptosis in WTRL1 cells increased gradually with the IH exposure time, and the apoptosis rate was the highest at $48 \mathrm{~h}(\mathrm{P}<0.05$; Fig. 6).

\section{Discussion}

OSA is characterized by intermittent airway obstruction and systemic hypoxia during sleep. OSA affects $5-25 \%$ of the general population. However, previous studies have illustrated a rising prevalence of moderate-to-severe sleep disordered breathing in men (ranging from 10-49\%, depending on age and cohort) and women (ranging from 10-23\%) in western countries $(20,21)$. OSA frequently goes unnoticed by people who are at highest risk for developing the disease (22-25). OSA is recognized as a low-grade systemic inflammatory disease; it induces inflammatory responses and can be an indication of metabolic deregulation, in addition to posing a great risk for apoptotic cell death (26). Apoptosis can be triggered by two distinct signalling pathways, namely, the intrinsic and extrinsic pathways $(27,28)$. The intrinsic apoptotic pathway is elicited by a wide range of intracellular stress conditions, including cytokine deprivation, DNA damage, oxidative stress, cytosolic $\mathrm{Ca}^{2+}$ overload and endoplasmic reticulum 
A

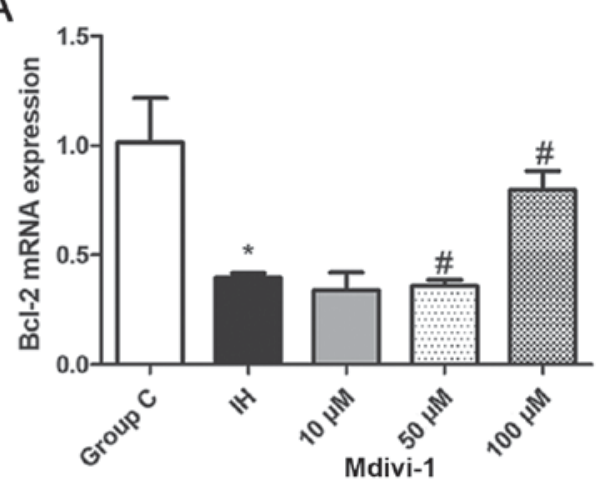

$\mathrm{C}$

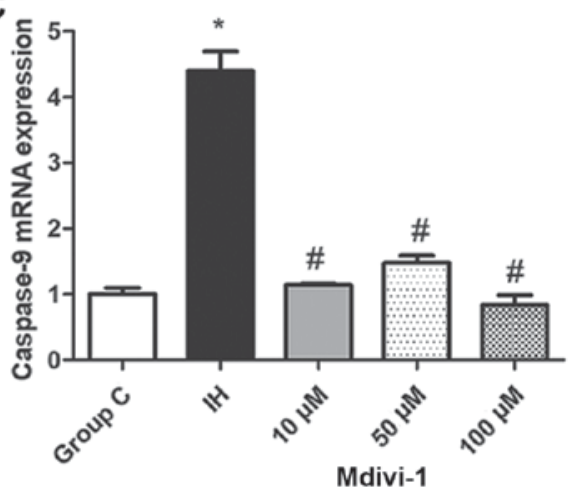

B
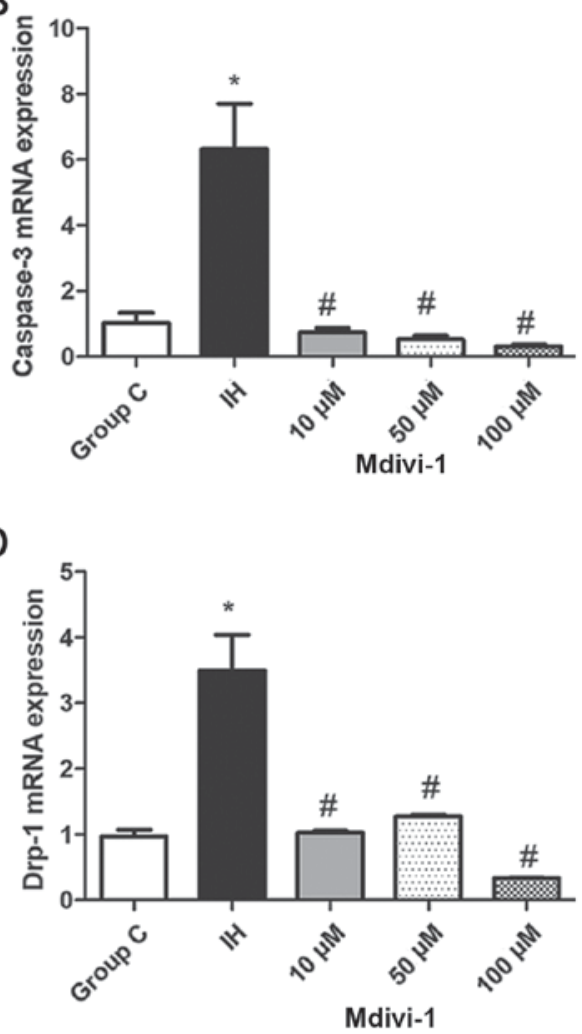

E

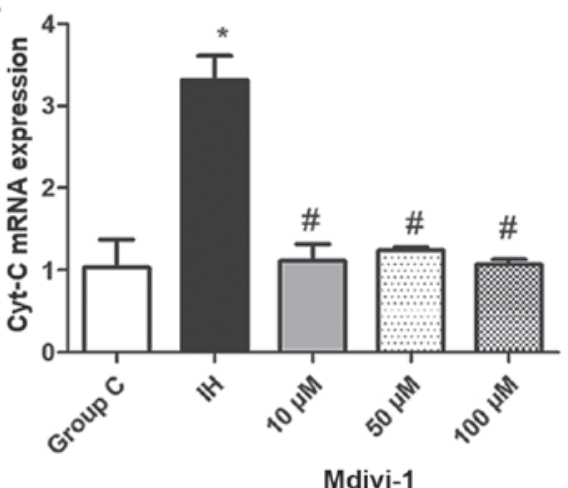

Figure 3. Detection of Bcl-2, caspase-3, caspase-9, Drp-1 and Cyt-C expression levels in WTRL1 cells via reverse transcription-quantitative polymerase chain reaction. (A) Bcl-2 levels. (B) Caspase-3 levels. (C) Caspase-9 levels. (D) Drp-1 levels. (E) Cyt-C levels. Compared with those in group C, Bcl-2 mRNA levels were lower, but caspase-3 and caspase- 9 mRNA levels were higher in the IH group, and these differences were statistically significant "P<0.05 vs. group C. Compared with those in the IH group, Bcl-2 mRNA expression levels were increased and caspase-3, and Drp-1 mRNAs were decreased After Mdivi-1 intervention, "P<0.05 vs. the IH group. IH, intermittent hypoxia; Mdivi, mitochondrial division inhibitor-1; Bcl, B-cell lymphoma; Cyt-c, cytochrome C; group C, control group.

stress. In the extrinsic pathway, apoptosis is triggered by death receptors, including FAS-associated death domain protein, therefore activating caspases-8 and 10 (the initiator caspases), which in turn activate executioner caspases-3, 6 and 7. In the intrinsic pathway, the mitochondrial permeability transition pore serves a pivotal role in regulating the release of pro-apoptotic proteins, including Cyt-C. The Cyt-C released from mitochondria initiates apoptosome assembly, activating factor 1 and caspase-9, an initiator caspase that cleaves and activates caspases-3 and 7 (29).

IH causes mitochondrial and endoplasmic reticulum system damage and produces inflammatory factors that cause lung injury. Sustained and IH-induced cell apoptosis mediates the mitochondrial apoptosis signalling pathway (30). A number of hypoxia and inflammatory factors activate caspases via the mitochondrial apoptosis signalling pathway. Mitochondrial morphology is now recognized as an important determinant of the energetic state of mitochondria. IH/hypercapnia leads to oxidative stress due to the mitochondrial response. Mitochondria constantly undergo fusion and fission, which are essential for organelle fidelity. Lung cell apoptosis occurs through a highly regulated proteolytic process that eliminates unwanted, damaged, or altered cells. However, abnormal mitochondrial fission is involved in regulating apoptosis (31).

Mdivi-1 has emerged as a critical regulator of cellular function and differentiation under hypoxic conditions. However, the role of mitochondrial dynamics in hypoxia-induced angiogenesis remains to be elucidated. The aim of the present 
A

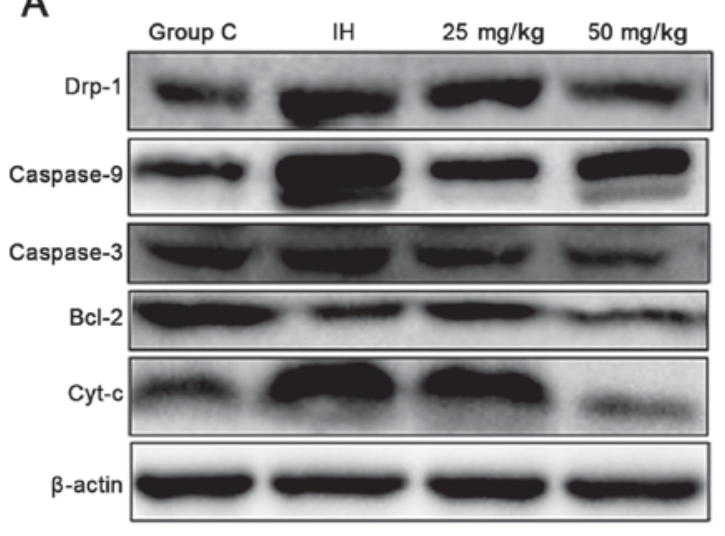

C

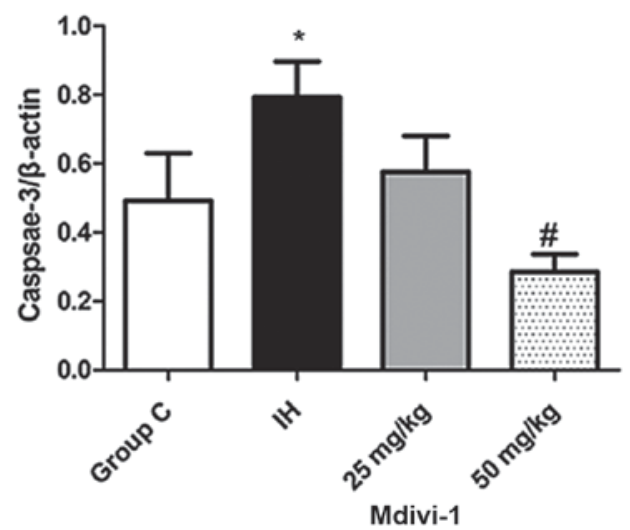

E

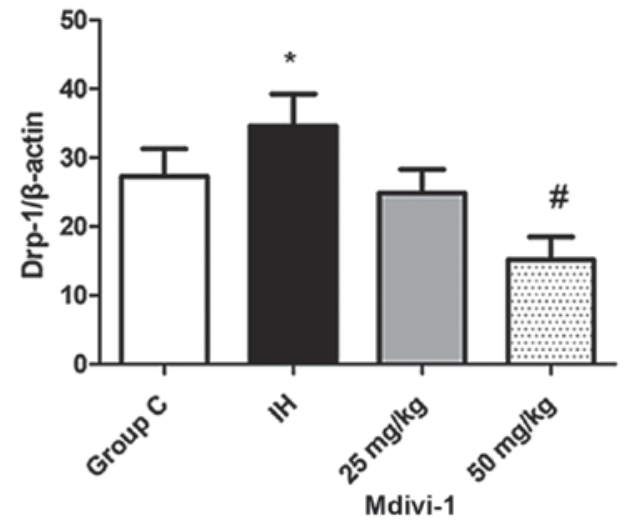

B

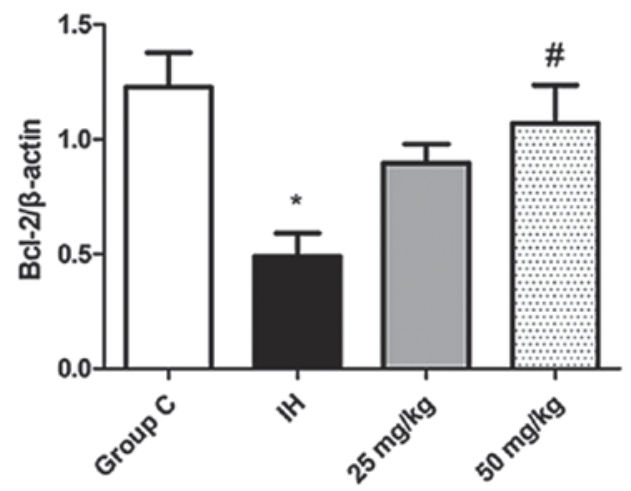

Mdivi-1

D

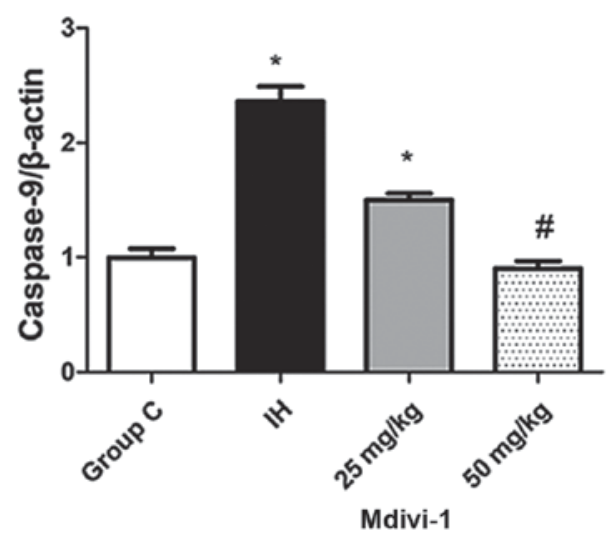

F

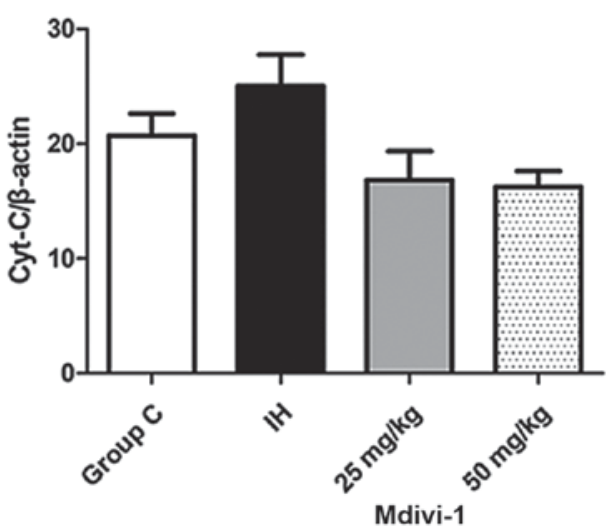

Figure 4. Detection of Bcl-2, caspase-3, caspase-9, Cyt-C and Drp-1 protein expression levels in rat lungs via western blotting assays. (A) (Lane 1) Group C, n-8. (Lane 2) IH group, $\mathrm{n}=8$. (Lane 3) $\mathrm{IH}+25 \mathrm{mg} / \mathrm{kg}$ Mdivi-1 group, $\mathrm{n}=8$. (Lane 4) $\mathrm{IH}+50 \mathrm{mg} / \mathrm{kg}$ Mdivi-1 group, $\mathrm{n}=8$. Compared with that in the group C, the protein expression of (B) Bcl-2 in the IH group was decreased and that of (C) caspase-3, (D) caspase-9 and (E) Drp-1 was increased in the IH group. (F) The changes in protein expression of $\mathrm{Cyt} \mathrm{C}$ were not significant. ${ }^{*} \mathrm{P}<0.05$ vs. group $\mathrm{C}$. Compared with the IH group, the protein expression of $\mathrm{Bcl}-2$ was increased and caspase- 3 , caspase- 9 , and Drp- 1 was decreased in the IH+50 mg/kg Mdivi- 1 group, ${ }^{\# P}<0.05$ vs. IH. IH, intermittent hypoxia; Mdivi, mitochondrial division inhibitor-1; Bcl, B-cell lymphoma; Cyt-c, cytochrome C; group C, control group.

study was to investigate the protective effect and possible mechanism of Mdivi-1 in lung cell apoptosis induced by IH.

Several studies have revealed that Drp-1 participates in mitochondrial fission and the subsequent apoptosis induced by various stimuli $(32,33)$. Drp-1 assembles from the cytosol onto mitochondria at focal sites of mitochondrial fission; emerging evidence indicates that it is concomitantly involved in apoptosis with mitochondrial outer membrane permeabilization and that Drp-1 inhibition prevents partially intrinsic apoptosis $(34,35)$. The activity of the prototypical $\mathrm{Bcl}-2$ protein is usually considered anti-apoptotic (36). The Bcl-2 family is classified into three subgroups: Pro-apoptotic proteins (Bax, BAD, Bak and Bok); anti-apoptotic proteins (Bcl-2, Bcl-xL and Bcl-w) and BH3-only members [Bad, Bid, Bim, Noxa and PUMA (a p53-upregulated modulator of apoptosis)]. The caspase family of cysteine-aspartic proteases serves an important role in the initiation and completion of cell apoptosis and executes cellular apoptosis. For instance, caspase- 3 is the primary activator of apoptotic DNA fragmentation. Certain reports have demonstrated that the pro-apoptotic signalling 
A

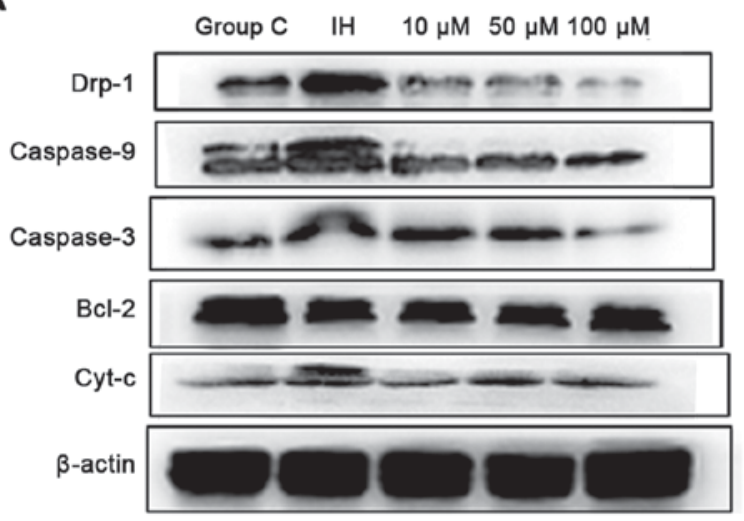

C

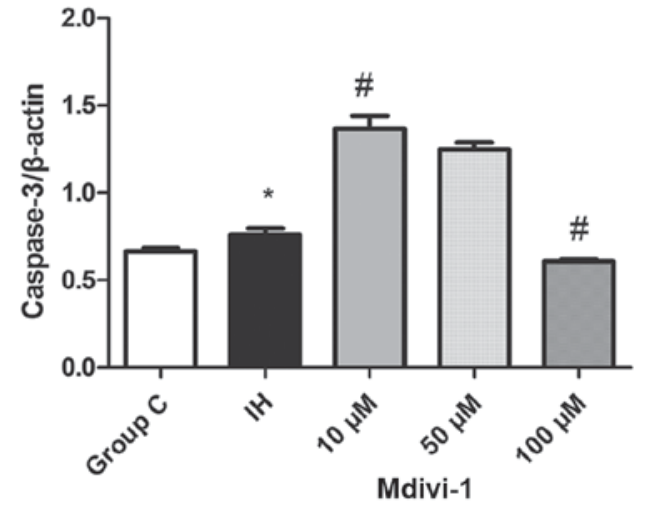

$E$

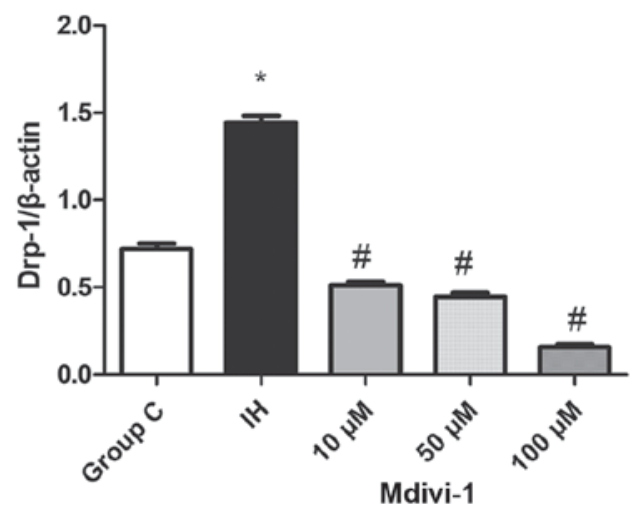

B

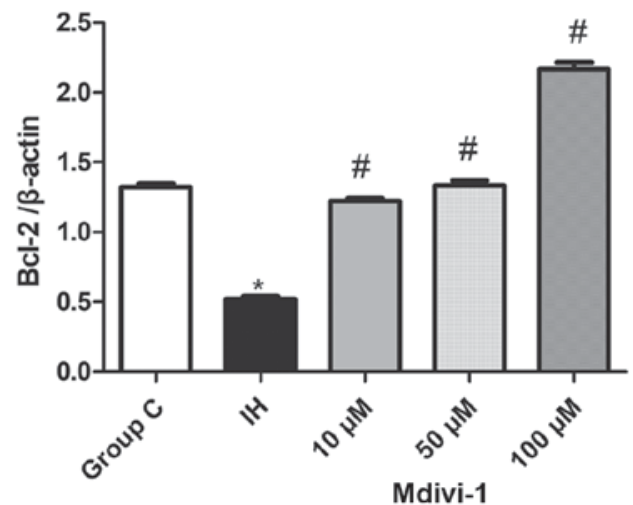

D

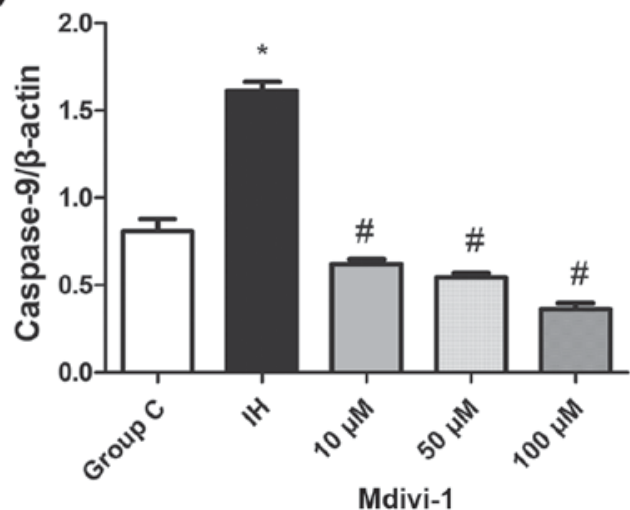

$\mathrm{F}$

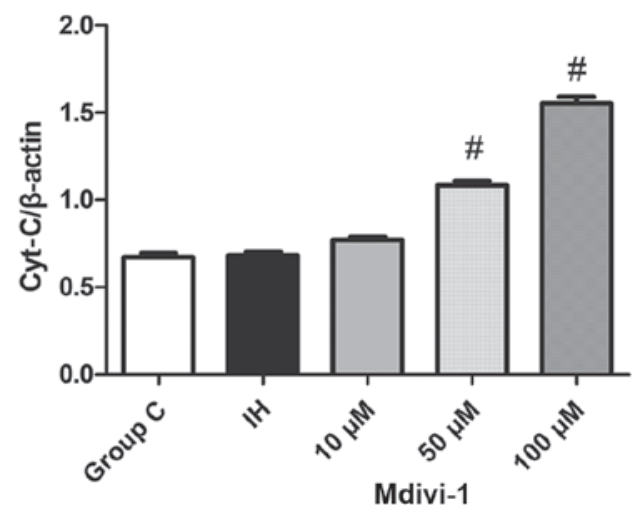

Figure 5. Detection of Bcl-2, caspase-3, caspase-9, Cyt-C and Drp-1 expression levels in WTRL1 cells via western blotting assays. (A) (Lane 1) Group C, $\mathrm{n}-8$. (Lane 2) $\mathrm{IH}$ group, $\mathrm{n}=8$. (Lane 3) $\mathrm{IH}+10 \mu \mathrm{M}$ Mdivi-1 group, $\mathrm{n}=8$. (Lane 4) $\mathrm{IH}+50 \mu \mathrm{M}$ Mdivi-1 group, $\mathrm{n}=8$. (Lane 5) IH+100 $\mu \mathrm{M} \mathrm{Mdivi}-1$ group, $\mathrm{n}=8$. Compared with the group C, (B) Bcl-2 protein expression in the IH group was decreased, but (C) caspase-3, (D) caspase-9 and (E) Drp-1 protein expression was increased, "P<0.05 vs. group C. Compared with the IH group, Bcl-2 protein expression was increased and caspase-9 Drp-1 and (F) Cyt-C protein expression was decreased in the IH+100 $\mu \mathrm{M}$ Mdivi-1 group, ${ }^{\#} \mathrm{P}<0.05$ vs. IH. Mdivi, mitochondrial division inhibitor-1; Drp-1, dynamin-related protein 1; IH, intermittent hypoxia; Bcl, B-cell lymphoma; group C, Control group.

of $\mathrm{Cyt}-\mathrm{C}$ triggers the caspase cascade amplification reaction, which leads to the cleavage of a set of proteins $(37,38)$.

Mitochondria are autonomous and morphologically dynamic organelles that structurally reflect a precise balance of ongoing fission and fusion within a cell. In the two models, in vitro and in vivo, it was observed that Mdivi-1 decreases mitochondrial fission and inhibits cell apoptosis in the lungs. These results agree with evidence from previous studies $(39,40)$. In this study, it was demonstrated that inhibiting mitochondrial fission by Mdivi-1 prevents lung apoptosis during IH and it was demonstrated that IH induced mitochondrial fission and apoptosis via a mitochondrial mechanism involving the transcriptional activation of caspase-3, caspase-9 and Drp-1. The results of the present study provide a well-characterized mechanism explaining how IH stimulates mitochondrial fission and consequent apoptosis, which may offer a novel therapeutic strategy for treating OSA via the mitochondrial apoptosis pathway. 
A

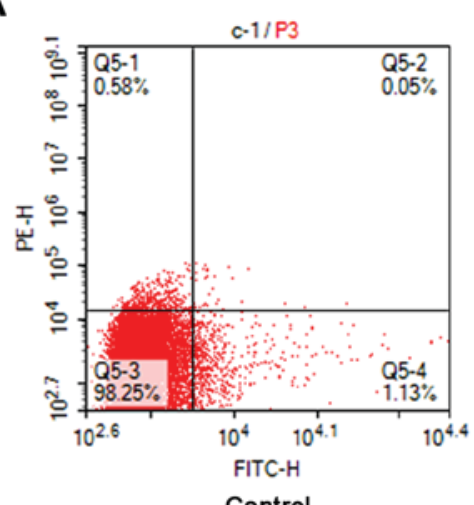

D

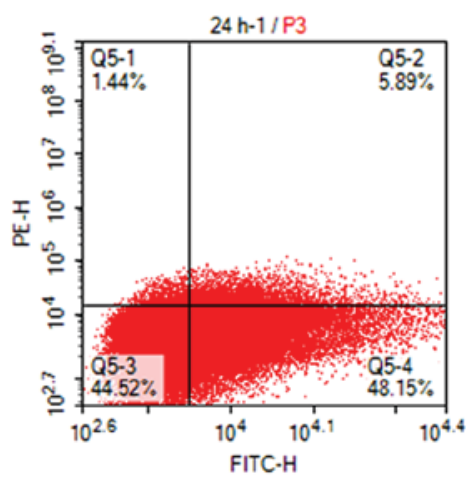

B

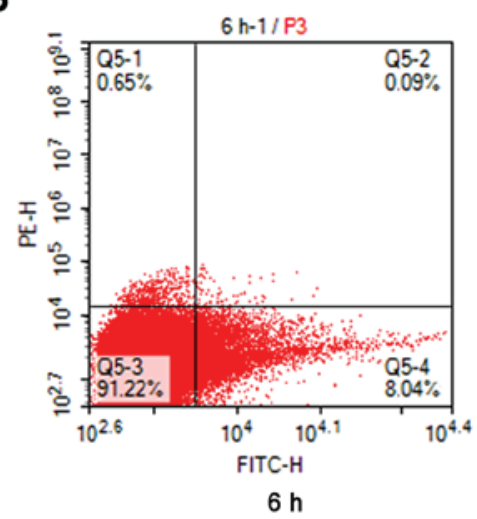

E

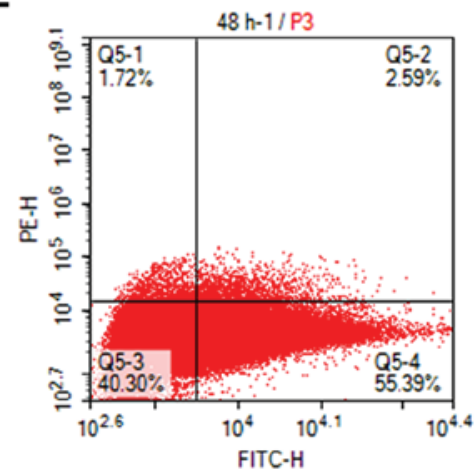

C

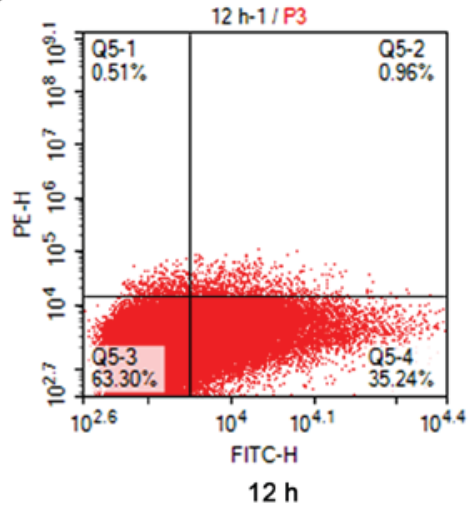

F

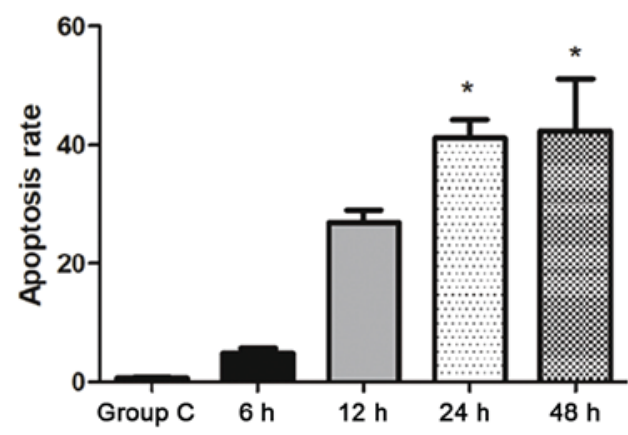

Figure 6. Detection of the apoptosis rate via flow cytometry. (A) Group C, n=8. (B) IH for $6 \mathrm{~h}, \mathrm{n}=8$. (C) IH for $12 \mathrm{~h}$, n=8. (D) IH for $24 \mathrm{~h}$, n=8. (E) IH for $48 \mathrm{~h}, \mathrm{n}=8$. (F) Detection of the apoptosis rate via flow cytometry. The apoptosis rate of WTRL1 cells increased gradually with the time exposed to IH and the apoptosis rate was the highest at $48 \mathrm{~h}$. ${ }^{*} \mathrm{P}<0.05$ vs. group $\mathrm{C}$. $\mathrm{IH}$, intermittent hypoxia; group $\mathrm{C}$, control group.

\section{Acknowledgements}

Not applicable.

\section{Funding}

The present study was funded by the Youth Fund Project of Guizhou Provincial People's Hospital (GZSYQN [2016] grant no. 16); the Technology Project of Guizhou Province (contract [2016] No. 2907); the Major Disease Prevention and Control Research of Guizhou Province (contract [2015] grant no. 78); the Science and Technology Project of a tobacco corporation (Guizhou Province division) of China (contract [201608]).

\section{Availability of data and materials}

The datasets used and/or analysed in the present study are available from the corresponding author upon reasonable request.

\section{Authors' contributions}

DZ and XY contributed to the conception and design of the study. CY and ZW collected the data and revised the manuscript for important intellectual content. DZ and XZ analysed and interpreted the data and drafted the manuscript. All authors read and approved the final manuscript.

\section{Ethics approval and consent to participate}

All procedures performed in studies involving animals were in accordance with the ethical standards of the institution or practice at which the studies were conducted. The study was approved by the Ethics Committee of Guizhou Provincial People's Hospital.

\section{Patient consent for publication}

Not applicable.

\section{Competing interests}

The authors declare that they have no competing interests.

\section{References}

1. Young T, Evans L, Finn L and Palta M: Estimation of the clinically diagnosed proportion of sleep apnea syndrome in middle-aged men and women. Sleep 20: 705-706, 1997.

2. Punjabi NM: The epidemiology of adult obstructive sleep apnea. Proc Am Thorac Soc 5: 136-143, 2008.

3. Sakao S, Taraseviciene-Stewart L, Lee JD, Wood K, Cool CD and Voelkel NF: Initial apoptosis is followed by increased proliferation of apoptosis-resistant endothelial cells. FASEB J 19: 1178-1180, 2005.

4. Haslip M, Dostanic I, Huang Y,Zhang Y, Russell KS, Jurczak MJ, Mannam P, Giordano F, Erzurum SC and Lee PJ: Endothelial Ucp2 regulates mitophagy and pulmonary hypertension during intermittent-hypoxia. Arterioscler Thromb Vasc Biol 35: 1166-1178, 2015. 
5. Zhou S, Yin X, Zheng Y, Miao X, Feng W, Cai J and Cai L: Metallothionein prevents intermittent hypoxia-induced cardiac endoplasmic reticulum stress and cell death likely via activation of Akt signaling pathway in mice. Toxicol Lett 227: 113-123, 2014.

6. Liu KX, Chen GP, Lin PL, Huang JC, Lin X, Qi JC and Lin QC: Detection and analysis of apoptosis- and autophagy-related miRNAs of mouse vascular endothelial cells in chronic intermittent hypoxia model. Life Sci 193: 194-199, 2018.

7. Ren J, Liu W, Deng Y, Li GC, Pan YY, Xie S, Jin M and Liu HG: Losartan attenuates aortic endothelial apoptosis induced by chronic intermittent hypoxia partly via the phospholipase C pathway. Sleep Breath 21: 679-689, 2017.

8. Wang J, Hansen K, Edwards R, Van Houten B and Qian W: Mitochondrial division inhibitor 1 (mdivi-1) enhances death receptor-mediated apoptosis in human ovarian cancer cells. Biochem Biophys Res Commun 456: 7-12, 2015.

9. Kim DY, Jung SY, Kim YJ, Kang S, Park JH, Ji ST, Jang WB, Lamichane S, Lamichane BD and Chae YC, et al Hypoxia-dependent mitochondrial fission regulates endothelial progenitor cell migration, invasion, and tube formation. Korean J Physiol Pharmacol 22: 203-213, 2018.

10. Gonder JC and Laber K: A renewed look at laboratory rodent housing and management. ILAR J 48: 29-36, 2007.

11. Shortt CM, Fredsted A, Chow HB, Williams R, Skelly JR, Edge D, Bradford A and O'Halloran KD: Reactive oxygen species mediated diaphragm fatigue in a rat model of chronic intermittent hypoxia. Experiment physiology 99: 688-700, 2014.

12. Briancon-Marjollet A, Monneret D, Henri M, Hazane-Puch F Pepin JL, Faure P and Godin-Ribuot D: Endothelin regulates intermittent hypoxia-induced lipolytic remodelling of adipose tissue and phosphorylation of hormone-sensitive lipase J Physiol 594: 1727-1740, 2016.

13. Poorhassan M, Navae F, Mahakizadeh S, Bazrafkan M, Nikmehr B, Abolhassani F, Ijaz S, Yamini N, Dashti N, Mehrannia K, et al: Flaxseed can reduce hypoxia-induced damages in rat testes. Int J Fertil Steril 12: 235-241, 2018

14. Krause BJ, Casanello P, Dias AC, Arias P, Velarde V, Arenas GA, Preite MD and Iturriaga R: Chronic intermittent hypoxia-Induced vascular dysfunction in rats is reverted by N-Acetylcysteine supplementation and arginase inhibition. Front Physiol 9: 901, 2018.

15. Park SW, Kim KY, Lindsey JD, Dai Y, Heo H, Nguyen DH, Ellisman MH, Weinreb RN and Ju WK: A selective inhibitor of drp1, mdivi-1, increases retinal ganglion cell survival in acute ischemic mouse retina. Invest Ophthalmol Vis Sci 52: 2837-2843, 2011.

16. Burkholder T, Foltz C, Karlsson E, Linton CG and Smith JM: Health evaluation of experimental laboratory mice. Curr Protoc Mouse Biol 2: 145-165, 2012

17. Kostopanagiotou G, Avgerinos E, Costopanagiotou C, Arkadopoulos N, Andreadou I, Diamantopoulou K, Lekka M, Smyrniotis V and Nakos G: Acute lung injury in a rat model of intestinal ischemia-reperfusion: The potential time depended role of phospholipases A(2) J Surg Res 147: 108-116, 2008.

18. Livak KJ and Schmittgen TD: Analysis of relative gene expression data using real-time quantitative PCR and the 2(-Delta Delta C(T)) method. Method 25: 402-408, 2001.

19. Nicole NL, Satriotomo I, Harrigan DJ and Mitchell GS: Acute intermittent hypoxia induced phrenic long-term facilitation despite increased SOD1 expression in a rat model of ALS. Exp Neurol 273: 138-150, 2015.

20. Peppard PE, Young T, Barnet JH, Palta M, Hagen EW and Hla KM: Increased prevalence of sleep-disordered breathing in adults. Am J Epidemiol 177: 1006-10014, 2013.

21. Heinzer R, Vat S, Marques-Vidal P, Marti-Soler H, Andries D, Tobback N, Mooser V, Preisig M, Malhotra A, Waeber G, et al: Prevalence of sleep-disordered breathing in the general population: The HypnoLaus study. Lancet Respir Med 3: 310-318, 2015

22. Drager LF, Togeiro SM, Polotsky VY and Lorenzi-Filho G: Obstructive sleep apnea: A cardiometabolic risk in obesity and the metabolic syndrome. J Am Coll Cardiol 62: 569-576, 2013.
23. Drager LF, Polotsky VY and Lorenzi-Filho G: Obstructive sleep apnea: An emerging risk factor for atherosclerosis. Chest J 140: 534-542, 2011

24. Sahlin C, Sandberg O, Gustafson Y, Bucht G, Carlberg B, Stenlund $\mathrm{H}$ and Franklin KA: Obstructive sleep apnea is a risk factor for death in patients with stroke: A 10-year follow-up. Arch Intern Med 168: 297-301, 2008.

25. Xie C, Zhu R, Tian Y and Wang K: Association of obstructive sleep apnoea with the risk of vascular outcomes and all-cause mortality: A meta-analysis. BMJ Open 7: e013983, 2017.

26. Zychowski KE, Sanchez B, Pedrosa RP, Lorenzi-Filho G, Drager LF, Polotsky VY and Campen MJ: Serum from obstructive sleep apnea patients induces inflammatory responses in coronary artery endothelial cells. Atherosclerosis 254: 59-66, 2016.

27. Galluzzi L, Vitale I, Abrams JM, Alnemri ES, Baehrecke EH, Blagosklonny MV, Dawson TM, Dawson VL, El-Deiry WS, Fulda S, et al: Molecular definitions of cell death subroutines: Recommendations of the nomenclature committee on cell death 2012. Cell Death Differ 19: 107-120, 2012.

28. Elmore S: Apoptosis: A review of programmed cell death. Toxicol Pathol 35: 495-516, 2007.

29. Mariño G, Niso-Santano M, Baehrecke EH and Kroemer G: Self-consumption: The interplay of autophagy and apoptosis. Nat Rev Mol Cell Biol 15: 81-94, 2014.

30. Douglas RM, Ryu J, Kanaan A, Del Carmen Rivero M, Dugan LL, Haddad GG and Ali SS: Neuronal death during combined intermittent hypoxia/hypercapnia is due to mitochondrial dysfunction. Am J Physiol Cell Physiol 298: C1594-C1602, 2012.

31. Li J, Donath S, Li Y, Qin D, Prabhakar BS and Li P: miR-30 regulates mitochondrial fission through targeting p53 and the dynamin-related protein-1 pathway. PLoS Genet 6: e1000795, 2010.

32. Chen Y, Lin JR and Gao PJ: Mitochondrial division inhibitor Mdivi-1 ameliorates angiotensin II-induced endothelial dysfunction. Sheng Li Xue Bao 68: 669-676, 2016.

33. Oettinghaus B, D'Alonzo D, Barbieri E, Restelli LM, Savoia C, Licci M, Tolnay M, Frank S and Scorrano L: DRP1-dependent apoptotic mitochondrial fission occurs independently of BAX, BAK and APAF1 to amplify cell death by BID and oxidative stress. Biochim Biophys Acta 1857: 1267-1276, 2016.

34. Tanaka A and Youle RJ: A chemical inhibitor of DRP1 uncouples mitochondrial fission and apoptosis. Mol Cell 29: 409-410, 2008.

35. Frank S, Gaume B, Bergmann-Leitner ES, Leitner WW, Robert EG, Catez F, Smith CL and Youle RJ: The role of dynamin-related protein 1, a mediator of mitochondrial fission, in apoptosis. Dev Cell 1: 515-525, 2001.

36. Czabotar PE, Lessene G, Strasser A and Adams JM: Control of apoptosis by the BCL-2 protein family: Implications for physiology and therapy. Nat Rev Mol Cell Biol 15: 49-63, 2014.

37. Di Pietro R and Zauli G: Emerging non-apoptotic functions of tumor necrosis factor-related apoptosis-inducing ligand (TRAIL)/Apo2L. J Cell Physiol 201: 331-340, 2004.

38. Sikora E, Bielak-Zmijewska A, Magalska A, Piwocka K, Mosieniak G, Kalinowska M, Widlak P, Cymerman IA and Bujnicki JM: Curcumin induces caspase-3-dependent apoptotic pathway but inhibits DNA fragmentation factor 40/caspase-activated DNase endonuclease in human Jurkat cells. Mol Cancer Ther 5: 927-934, 2006.

39. Han XJ, Yang ZJ, Jiang LP, Wei YF, Liao MF, Qian Y, Li Y, Huang X, Wang JB, Xin HB and Wan YY: Mitochondrial dynamics regulates hypoxia-induced migration and antineoplastic activity of cisplatin in breast cancer cells. Int J Oncol 46: 691-700, 2015.

40. Sanderson TH, Raghunayakula S and Kumar R: Neuronal hypoxia disrupts mitochondrial fusion. Neuroscience 301: 71-78, 2015. 